\title{
Abhandlungen
}

\author{
Hans-Peter Blossfeld
}

\section{Globalisierung, wachsende Unsicherheit und die Veränderung der Chancen der jungen Generation in modernen Gesellschaften}

\author{
Ausgewählte Ergebnisse des GLOBALIFE-Projekts
}

\begin{abstract}
Dieser Beitrag konzeptualisiert die Effekte des Globalisierungsprozesses zunächst mit Hilfe eines Mehrebenenmodells und stellt dann ausgewählte Ergebnisse des GLOBALIFE-Projekts dar. Er beschreibt, (1) wie sich die Globalisierung als makrostruktureller Prozess in ausgewählten modernen Ländern seit dem Beginn der siebziger Jahre des letzten Jahrhunderts entwickelt hat, (2) wie die durch Globalisierung erzeugte steigende Unsicherheit auf der Mesoebene durch Institutionen des Bildungs-, Beschäftigungs- und Wohlfahrtsstaatssystems auf bestimmte soziale Gruppen kanalisiert wird und (3) wie die Akteure auf der individuellen Ebene im Alltag in verschiedenen Ländern darauf reagieren. Die Darstellung ausgewählter Ergebnisse konzentriert sich auf die Phase der beruflichen Etablierung und auf die Veränderungen sozialer Ungleichheit bei jungen Erwachsenen in modernen Gesellschaften.
\end{abstract}

\section{$1 \quad$ Einleitung}

Der Prozess der Globalisierung ist sicherlich kein neues Phänomen, aber die Intensität und Reichweite grenzüberschreitender Interaktionsbeziehungen, seien es ökonomische Transaktionen, informationelle und kulturelle Austauschprozesse oder internationale politische Abmachungen und Verträge, scheinen seit der Mitte der achtziger Jahre, insbesondere seit dem Ende des Ost-West-Gegensatzes, in den meisten Industrieländern schubartig zugenommen zu haben (siehe Abbildung 2). Dieser Beitrag beschäftigt sich mit den Effekten dieser Ausweitung der gesellschaftlichen Beziehungen über die Grenzen des Nationalstaates hinaus auf den Wandel der individuellen Lebensläufe in verschiedenen modernen Gesellschaften. Er entwickelt Thesen zu der spezifischen Frage, wie sich die Bildungs- und Berufsverläufe von jungen Männern und Frauen in OECD-Gesellschaften im Globalisierungsprozess wandeln und berichtet über ausgewählte Ergebnisse des GLOBALIFE-Projekts zu den Veränderungen sozialer Ungleichheit bei jungen Erwachsenen in modernen Ländern. 


\section{Globalisierung und wachsende Unsicherheit in modernen Gesellschaften}

Die meisten Sozialforscher gehen heute davon aus, dass der Prozess der Globalisierung gekennzeichnet ist durch das Zusammenwirken von vier makrostrukturellen Entwicklungen, die sich seit der Mitte der achtziger Jahre zunehmend durchgesetzt haben (siehe Abbildung

1). Diese beinhalten:

(1) Die zunehmende Internationalisierung von Märkten und den damit verbundenen wachsenden Wettbewerb zwischen Ländern mit sehr unterschiedlichen Lohn- und Produktivitätsniveaus sowie verschiedenen Sozialstandards (insbesondere nach dem Fall des „Eisernen Vorhangs“ und der Integration asiatischer Länder in den Weltmarkt).

(2) Die Verschärfung des Standortwettbewerbs zwischen Sozialstaaten und die sich daraus ergebende Tendenz zur Senkung von Unternehmenssteuern in vielen Ländern sowie die zunehmende Neigung moderner Staaten zur Deregulierung, Privatisierung und Liberalisierung und damit zu einer Stärkung des Marktes als Koordinationsmechanismus.

(3) Die rasche weltweite Vernetzung von Personen, Unternehmen und Staaten auf der Grundlage neuer Informations- und Kommunikationstechnologien und - daraus resultierend - die zunehmende globale Interdependenz der Akteure sowie die wachsende Beschleunigung von sozialen und wirtschaftlichen Interaktionsprozessen.

(4) Den rasanten Bedeutungszuwachs von weltweit vernetzten Märkten und die damit verbundene zunehmende Interdependenz und Volatilität lokaler Märkte, die von schwer prognostizierbaren weltweiten sozialen, politischen und ökonomischen „externen Schocks“ und Ereignissen (wie z. B. Kriege, ökonomische Krisen, Verbrauchermoden, technologische Innovationen) immer stärker beeinträchtigt werden.

In den vergangenen Jahren hat die Globalisierung damit auf der einen Seite zu Produktivitätszuwächsen und zu einer allgemeinen Verbesserung des Lebensstandards in modernen Gesellschaften geführt. Aber auf der anderen Seite ist Globalisierung in diesen Ländern auch verbunden mit einer Zunahme unerwarteter Marktentwicklungen in einer sich immer schneller verändernden Weltwirtschaft, mit rapideren sozialen und ökonomischen Wandlungsprozessen, mit einer immer stärker abnehmenden Vorhersagbarkeit von ökonomischen und sozialen Entwicklungen und, daraus resultierend, mit einer zunehmenden allgemeinen Unsicherheit, verbunden mit einem wachsenden Bedarf an Flexibilität in den Unternehmen und Betrieben (siehe Abbildung 1).

In einer kürzlich veröffentlichten Untersuchung hat die Konjunkturforschungsstelle der Eidgenössischen Technischen Hochschule (ETH) in Zürich ein neues Instrument zur Messung des Globalisierungsgrades von Gesellschaften vorgelegt (Dreher 2005). Dieser Index ist für 123 Länder auf der Grundlage einer größeren Anzahl von Indikatoren für den Zeitraum von 1970 bis 2003 entwickelt worden und integriert wirtschaftliche, soziale, informationelle und politische Dimensionen der Globalisierung in einer Maßzahl. Zu den Indikatoren wirtschaftlicher Globalisierung gehören dabei (1) die Handels- und Investitionsströme in Relation zum BIP (Import- und Exportanteile, Auslandsdirektinvestitionen, kurzfristiges spekulatives Kapital, Einkommens-/Lohnzahlungen an Ausländer) sowie (2) der Abbau von Handelsbeschränkungen (verdeckte) Importbeschränkungen, Zölle, Besteuerung internationalen Handels, Kapitalverkehrsbeschränkungen). Zu den Indikatoren sozialer und informationeller Globalisierung gehören Informationen über (1) die wachsenden Beziehungsgeflechte (gemessen als Volumen der Auslandstelefongespräche, Transferleistungen (ohne 


\section{Abb. 1: Globalisierung und wachsende Unsicherheit in modernen Gesellschaften}

\section{GLOBALISIERUNG}

\author{
Internationalisierung \\ von $M$ ärkten \\ W ettbewerb zwischen \\ $L$ ändern mit \\ unterschiedlichen \\ Lohn- und \\ Produktivitätsniveaus \\ bzw. Sozialstandards
}

V erstärkter Standortwettbewerb zwischen

Sozialstaaten, Politik der Deregulierung, Privatisierung und Liberalisierung

\section{Zunehmende} weltweite V ernetzung durch neue

Informations- und

Kommunikations-

Technologien
B edeutungszuwachs von M ärkten, aber gleichzeitig zunehmende Instabilität und $V$ erwundbarkeit lokaler M ärkte durch externe weltweite Schocks wachsende G eschwindigkeit von Innovationen, beschleunigter sozialer und ökonomischer W andel
B eschleunigung der $M$ arktprozesse auf allen M ärkten
Zunahme unvorhersehbare $M$ arktentwicklungen, steigende $V$ olatilität von $M$ ärkten

\section{Z unehmende Unsicherheit}

\section{INSTITUTIONELLE FILTER}

B eschäftigungs-
systeme $\quad$ Bildungssyteme $\quad \begin{gathered}\text { Wohlfahrtsstaats- } \\ \text { regime }\end{gathered} \quad$ Familiensysteme

... kanalisieren die durch G lobalisierung erzeugte Unsicherheit in spezifischer $W$ eise und beeinflussen ...

- das A usmaß an B eschäftigungsund A rbeitsplatzstabilität

- das A usmaß an

Flexibilitit und

Sicherheit

- die Häufigkeit

verschiedener

Formen von

K arrieremobilitäten die A usgestal tung des sozialen Sicherungs-

systems, z.B.

des Berufseinstiegs sowie dessen Timing

- M öglichkeiten der

W eiterbildung und

des lebenslangen

Lernens
- V erfügbarkeit von

K inderbetreuungs-

möglichkeiten

- B eschäftigungsför-

dernde $\mathrm{M}$ aßnahme

- Rentensysteme
- die Prävalenz verschiedener Familienformen, Haushaltsund Erwerbsmuster - die A usgestal tung familialer Rollen - das A usmaß familialer Pflegeverantwortlichkeiten

INDIVIDUALEBE NE

Unsicherheit wird auf spezifische gesellschaftliche Gruppen kanalisiert! (z.B. Jugendliche/junge Erwachsene, Personen mit geringer Bildung)

Quelle: Eigene Darstellung 


\section{Abb. 2: Veränderung des Globalisierungsgrades (Globalisierungsindex)}

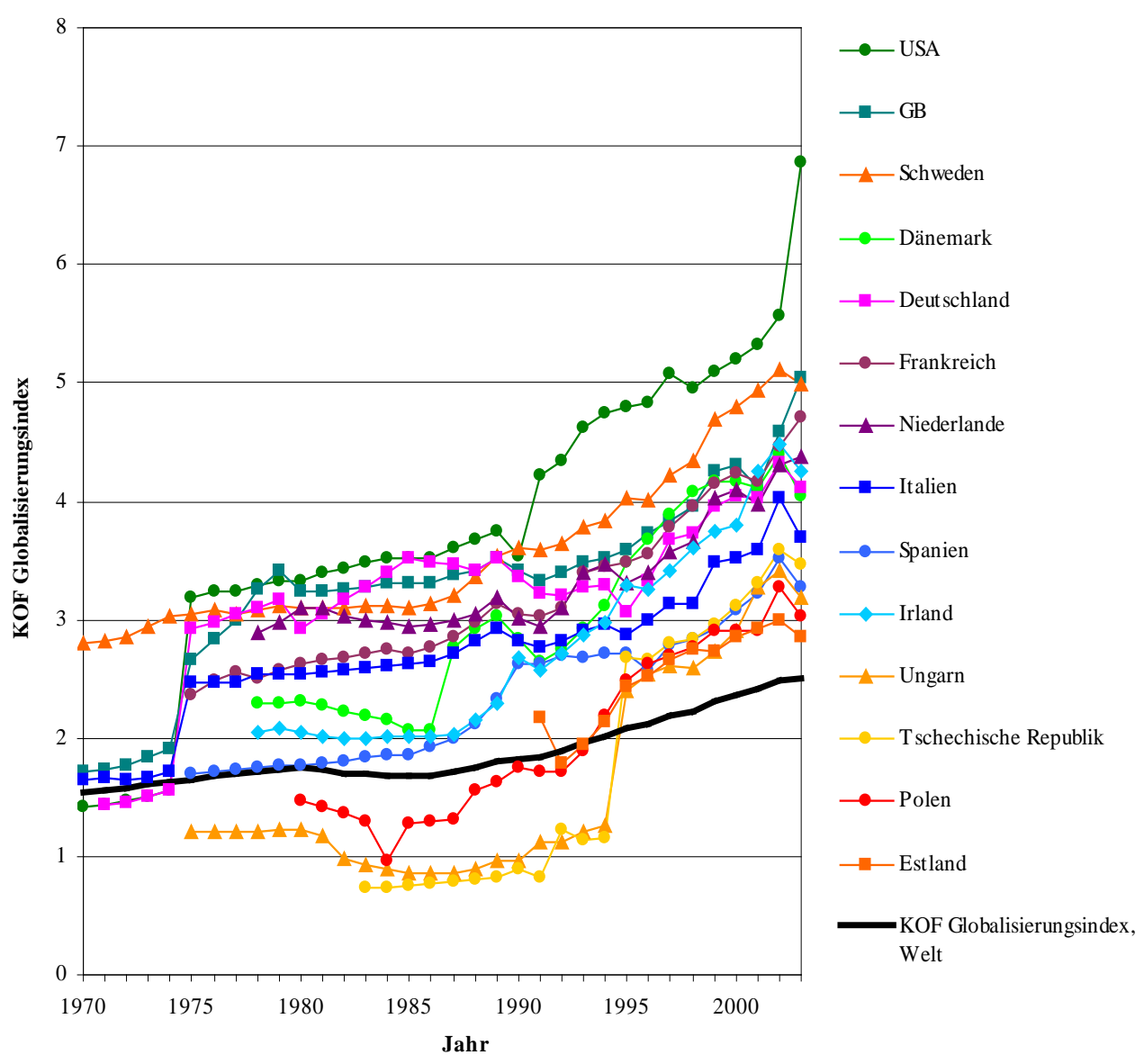

Gegenleistung), internationaler Tourismus, Durchschnittskosten eines Telefonats in die USA und Anteil der Ausländer an der Gesamtbevölkerung), (2) die Informations- und Kommunikationsflüsse (gemessen als Anzahl der Telefonanschlüsse (je 1000 Einwohner), Internet-Hosts (pro Einwohner), Internetnutzer (als Anteil an der Bevölkerung), Kabelfernsehen (je 1.000 Einwohner), Tageszeitungen (je 1.000 Einwohner), Radioempfänger (je 1.000 Einwohner) und (3) die „Amerikanisierung“ (gemessen als Anzahl der McDonalds-Restaurants je 100.000 Einwohner). Zu den Indikatoren politischer Globalisierung zählen schließlich die Anzahl der Botschaften im eigenen Land, die Mitgliedschaft in internationalen Organisationen und die Teilnahme an UN-Sicherheitsratsmissionen.

Abbildung 2 stellt die Veränderung der Globalisierungsintensität in den wichtigsten vom GLOBALIFE-Projekt untersuchten OECD-Ländern mit Hilfe dieser Maßzahl dar. Es wird dort deutlich, dass die liberalen Länder wie USA, Großbritannien und Irland sowie die skandinavischen Länder wie Schweden und Dänemark heute zu den am meisten globalisierten Ländern der Welt zählen. Dann kommen kontinentaleuropäische Länder wie Deutschland, Frankreich und die Niederlande. Erst danach findet man die südeuropäischen Länder wie Italien 
und Spanien und schließlich die ehemals sozialistischen Länder wie Ungarn, Tschechische Republik, Polen und Estland. Im Vergleich zum weltweiten Durchschnittsindex über alle 123 Länder (siehe dazu den KOF Globalisierungsindex, Welt, in Abbildung 2) wird deutlich, dass sich der Globalisierungsprozess in den europäischen Ländern und in den USA seit der Mitte der achtziger Jahre des vergangenen Jahrhunderts besonders intensiv vollzieht und dort die Dynamik der Entwicklung weit über dem globalen Durchschnittsniveau liegt.

\section{$3 \quad$ Globalisierung und der Umgang mit wachsender Unsicherheit auf der individuellen Ebene}

Wie das GLOBALIFE-Projekt ausführlich zeigt (siehe unten), führt die wachsende makrostrukturelle Unsicherheit im Zuge der Globalisierung zu einer Reihe von Veränderungen und Reaktionen auf der Ebene einzelner Handelnder im Alltag:

(1) Es fällt den Akteuren in modernen Gesellschaften in zunehmendem Maße schwer, kalkulierende, rationale Entscheidungen, insbesondere langfristig bindende Lebenslaufentscheidungen zu treffen, da sowohl die Verfügbarkeit von Handlungsalternativen als auch die Vorhersehbarkeit ihrer Folgen und Nebenwirkungen in den globalisierten Gesellschaften zunehmend unklarer wird. Aufgrund dieser zunehmenden Unsicherheit möglicher Handlungsergebnisse gewinnen im Globalisierungsprozess paradoxerweise gerade die lokalen Routinen, Faustregeln sowie regionalen Traditionen und Normen als Orientierungshilfen für Individuen und ihre Entscheidungen an Bedeutung. Damit ergibt sich auf der Handlungsebene über diese Mechanismen wieder eine starke Pfadabhängigkeit nationaler oder regionaler Entwicklungsprozesse im Globalisierungsprozess (siehe die Argumentation weiter unten).

(2) Insbesondere langfristige (Lebenslauf-)Entscheidungen werden in einer globalisierten Welt zunehmend schwieriger, so dass sich sozial und ökonomisch vor allem eine Verschiebung zugunsten einer an kurzfristigen Zeithorizonten orientierten Planung ergibt. Beispielsweise müssen am Aktienmarkt notierte Unternehmen heute in immer kürzeren Abständen Betriebsergebnisse (z. B. Quartalsberichte) vorlegen und es kommt zu einer systematischen Unterbewertung langfristiger Interessen von Betrieben und von in Unternehmen beschäftigten Arbeitskräften.

(3) Der in den vergangenen Jahren in verschiedenen Ländern zu beobachtende teilweise Abbau des Wohlfahrtsstaates verstärkt diese „Verunsichungs“-Tendenzen zusätzlich noch, da auch hier durch die Reduzierung wohlfahrtsstaatlicher Leistungen ehemalige Sicherheiten nach und nach brüchig werden.

(4) Darüber hinaus führt Globalisierung zu einer bedeutsamen Verschiebung der Machtkonstellationen am Arbeitsmarkt. „Verhandlungsstarke“ Gruppen, zumeist Arbeitgeber, verlagern in zunehmendem Maße ihre Marktrisiken auf bestimmte „,verhandlungsschwächere“ Arbeitnehmergruppen. Diejenigen Personen, die nicht fest im Erwerbsleben verankert sind (wie etwa die Berufseinsteiger oder Frauen, die nach einer familiären Erwerbsunterbrechung eine Beschäftigung suchen) bzw. sich an den Rändern des Arbeitsmarktes oder in unsicheren Beschäftigungsverhältnissen befinden, sind besonders von der Verlagerung dieser Marktrisiken zu ihren Ungunsten betroffen (siehe z. B. die Ergebnisse unten in Tabelle 1).

Zusammengenommen führen die Globalisierungsprozesse zu einer Verstärkung sozialer Ungleichheiten in westlichen Industriegesellschaften. Wie das GLOBALIFE-Projekt zeigt, 
werden aufgrund der zunehmenden Bedeutung von (Arbeits-)Märkten individuelle Arbeitsmarktressourcen (wie etwa Bildung, berufliche Qualifikation oder Berufserfahrung) und individuelle Merkmale (wie Geschlecht, ethnische Zugehörigkeit oder Lebensalter) für den Erwerbsverlauf zunehmend wichtiger und verstärken damit die bereits durch das Bildungssystem angelegten Ungleichheiten in den modernen Gesellschaften.

Das GLOBALIFE-Projekt zeigt schließlich, dass es durch zunehmende Unsicherheiten auch insgesamt schwerer wird, Vertrauensbeziehungen aufzubauen und aufrechtzuerhalten. Vertrauensbeziehungen beruhen im Allgemeinen auf der Bereitschaft, sich selbst langfristig an jemanden oder etwas zu binden, gleichzeitig aber auch in einen langfristig orientierten Austausch von Leistungen einzutreten (Eltern sorgen für ihre Kinder und erwarten, dass sie von ihren Kindern im Alter unterstützt werden; Partner, z. B. Frauen, treten in Vorleistung und erziehen die Kinder und bauen darauf, dass die Beziehung dauerhaft ist und sie später Unterstützung von ihrem Partner erhalten; sowohl Arbeitnehmer als auch Arbeitgeber vertrauen darauf, dass ihre Abmachungen auch langfristig eingehalten werden; Staatsbürger zahlen Arbeits-, Kranken- und Rentenversicherungsbeiträge und erwarten, dass sie staatliche Hilfe bekommen, wenn sie diese aufgrund von Arbeitslosigkeit, Krankheit oder Alter benötigen). Unter den Bedingungen steigender Unsicherheit in globalisierten Gesellschaften werden solche Versprechen jedoch immer problematischer, da häufiger unvorhergesehene Ereignisse auftreten, die dazu führen, dass die Gegenleistungen nicht mehr oder nicht mehr im versprochenen Umfang erbracht werden. Es kommt häufiger zu Enttäuschungen. Die Folge ist ein zunehmender Vertrauensverlust und eine Abnahme der Sicherungsfunktion von Familie, Verwandtschaft, Betrieb und Wohlfahrtsstaat in globalisierten Gesellschaften.

In der Phase des Übergangs vom Jugendlichen zum Erwachsenen erhalten langfristig bindende Entscheidungen einen besonders paradoxen Charakter (siehe unten): Zum einen sind sie sinnvoll, um Sicherheit für sich selbst und Glaubwürdigkeit sowie Vertrauen im Handeln mit anderen zu produzieren (z. B. die Heiratsentscheidung oder die Entscheidung für ein Kind), zum anderen sinkt damit gleichzeitig die Flexibilität und der Optionsspielraum im weiteren Lebenslauf mit Blick auf die sich immer rascher ändernden Anforderungen durch den globalen Wandel.

\section{Filterung des Globalisierungsprozesses durch länderspezifische Institutionen}

Ein zentrales Ergebnis des GLOBALIFE-Forschungsprojektes ist, dass die beschriebenen gemeinsamen globalen Wandlungsprozesse in verschiedenen Länderkontexten jedoch nicht zu dem gleichen Ergebnis führen müssen. Vielmehr trifft der Globalisierungsprozess in unterschiedlichen Länderkontexten auf verschiedene, fest verankerte institutionelle Strukturen, etwa wohlfahrtsstaatliche Einrichtungen oder bestimmte Formen der Regulierung von Arbeitsmärkten oder lokale Normen und Werte, die den Globalisierungsprozess in spezifischer Weise filtern (vgl. Abbildung 1). Diese Institutionen wandeln sich deswegen zwar im Zuge des Globalisierungsprozesses, bleiben aber in der Regel weiter von spezifischer Bedeutung. Ziel des GLOBALIFE-Projektes war es, diese Veränderungen in verschiedenen Ländern empirisch vergleichend über mehrere Jahrzehnte zu analysieren und gegenüberzustellen.

Der vom GLOBALIFE-Projekt vertretene Ansatz geht dabei davon aus, dass der Globalisierungsprozess von vornherein als kontingent und im Plural gedacht werden muss, wobei $\mathrm{zu}$ vermuten ist, dass den historisch gewachsenen länderspezifischen institutionellen und 
sozialen Strukturen eine starke Beharrungstendenz zukommt. Die Dauerhaftigkeit länderspezifischer Differenzen kann theoretisch damit begründet werden, dass die Institutionensysteme und Sozialstrukturen moderner Gesellschaften ineinander verwobene Arrangements mit hoher interner Komplementarität sind. Sie lassen sich adäquat nur in ihrer Gesamtheit als „länderspezifische Institutionenpakete“ begreifen. Beispielsweise sind die Bildungs- und Beschäftigungssysteme moderner Gesellschaften sehr eng miteinander verzahnt, wenn es um die Rolle der Lerninhalte in der Schule, die Organisationsformen der Bildungsinstitutionen, die Funktion der Bildungszertifikate für den Berufseinstieg, den Zusammenhang zwischen beruflichen Ausbildungsinstitutionen und Mobilitätsprozessen, die Herausbildung von Arbeitsmarktsegmenten oder die Intensität beruflicher Abstiegsprozesse geht. Ähnlich enge komplementäre Zusammenhänge wie zwischen dem Bildungs- und Beschäftigungssystem bestehen auch zwischen dem Bildungs- und Familiensystem, dem Arbeitsmarkt und den familiären Strukturen oder dem Beschäftigungssystem, dem Wohlfahrtsstaat und dem Familiensystem. Daraus folgt, dass die gesellschaftlichen Institutionen sich im Globalisierungsprozess adäquat nur in ihrem wechselseitigen Abhängigkeitsverhältnis empirisch analysieren lassen.

Für die einzelnen Nationalstaaten muss man also von eigenständigen Entwicklungslogiken im Globalisierungsprozess ausgehen. Die Nationalstaaten sind aber nicht als isolierte Entitäten zu begreifen, sondern als Komponenten größerer sich herausbildender internationaler Systeme, die über Weltmärkte, technologische Diffusionsprozesse, politische Vernetzung sowie Informations- und Kommunikationssysteme in einen immer intensiveren Austausch treten. Im Hinblick auf diesen Austausch zeigt sich, dass der Globalisierungsprozess zwar die räumlichen Unsicherheiten (durch weltweite Informations-, Kommunikations- und Transportmöglichkeiten) vermindert, dabei aber gleichzeitig vor allem die zeitbezogenen Unwägbarkeiten enorm vergrößert (siehe Abbildung 1).

\section{$5 \quad$ Mobilitätsmechanismen im Globalisierungsprozess}

Die Beschleunigung des Wandels auf den weltweit sich vernetzenden Märkten und die steigende Geschwindigkeit technologischer Innovationen auf globaler Ebene verändern die Verteilung der Berufe sowie die Struktur der Wirtschaftssektoren in den modernen, „lernenden“ Gesellschaften in immer schnellerer Folge. Dabei kann der Strukturwandel im Beschäftigungssystem durch fünf fundamentale Mobilitätsereignisse herbeigeführt werden: (1) durch den Eintritt junger Arbeitskräfte in den Arbeitsmarkt, (2) durch Mobilitätsentscheidungen jener, die bereits beschäftigt sind, (3) durch das Ausscheiden und das Wiedereintreten von Arbeitskräften während des Karriereverlaufs (z. B. aufgrund von Arbeitslosigkeit oder Krankheit), (4) durch Erwerbsunterbrechungen und den Wiedereintritt von Frauen im Rahmen des Familienzyklus und (5) durch die Verrentung sowie anderes langfristiges Ausscheiden aus dem Beschäftigungssystem.

Insgesamt zeigen die Analysen des GLOBALIFE-Projekts, dass der Strukturwandel des Beschäftigungssystems im Globalisierungsprozess dazu führt, dass Arbeitskräfte aus obsoleten Berufen und Wirtschaftssektoren in moderne, expandierende strömen. Aber die Arbeitskräfte, die aus den obsoleten Berufen, Firmen oder Industrien kommen, sind nicht notwendigerweise auch die gleichen, die die Positionen in den expandierenden Sektoren übernehmen. Diese Arbeitskräfte können rasch auf neue berufliche Tätigkeiten wechseln, oder sie können für eine kurze oder längere Zeit arbeitslos werden. Die Kontraktion eines Wirtschaftszweigs durch Frühpensionierung älterer Arbeitnehmer wird dagegen überhaupt 
keine Berufsmobilität zur Folge haben. In ähnlicher Weise kann die Expansion von Berufen oder Sektoren durch berufliche Mobilitätsprozesse oder durch den Eintritt aus den Reihen bisher nicht Erwerbstätiger (z. B. Berufsanfänger, Arbeitslose oder Frauen nach einer familiären Erwerbsunterbrechung) geschehen. Mit anderen Worten, der berufsstrukturelle und sektorale Wandel im Beschäftigungssystem kann sich in den modernen Ländern über sehr verschiedene Mobilitätsereignisse vollziehen und sich sehr unterschiedlich auf die Lebensverläufe aufeinander folgender Kohorten auswirken. Die Ergebnisse des GLOBALIFE-Projekts zeigen, dass in verschiedenen Ländern die historisch gewachsenen Institutionen nicht nur den Prozess des Entstehens und Vernichtens beruflicher Positionen im Globalisierungsprozess bestimmen, sondern auch die Richtung und das Ausmaß der verschiedenen Mobilitätsströme. Auf der Handlungsebene wirken diese institutionellen Strukturen moderner Gesellschaften dabei als Anreize oder Hindernisse für organisatorische Anpassungen von Arbeitgebern und Mobilitätsentscheidungen von Individuen.

Wir wollen im folgenden drei für das GLOBALIFE-Projekt zentrale länderspezifische Mobilitätsmechanismen kurz diskutieren, die sich aus Unterschieden (1) in den Bildungssystemen, (2) den industriellen Beziehungen zwischen Arbeit und Kapital und (3) den Wohlfahrtsstaaten ergeben.

\subsection{Differenzen in den Bildungssystemen}

Die allgemeine und berufliche Ausbildung sowie die Weiterbildung wird in modernen Gesellschaften sehr vielfältig organisiert. Diese Organisationsformen haben weit reichende Implikationen für den Zeitpunkt des Berufseintritts, die Art und Weise, wie in einer Gesellschaft Arbeitsplätze mit Arbeitskräften besetzt werden und die Fähigkeit der Arbeitskräfte und Arbeitsorganisationen, sich auf neue Anforderungen im Globalisierungsprozess flexibel und „lernend“ einzustellen.

Hinsichtlich des allgemeinen Bildungssystems sind zunächst vor allem nationale Unterschiede (1) in der Beziehung zwischen verschiedenen Schullaufbahnen zueinander (stark gegliedert vs. einheitlich, geschlossen vs. relativ offen) und (2) in den kohortenspezifischen Bildungsbeteiligungsraten in den verschiedenen Schultypen für das GLOBALIFE-Projekt interessant gewesen.

In Bezug auf das berufliche Bildungssystem muss man zwischen Ländern unterscheiden, die ihre Ausbildung vor allem in Form schulischer Ausbildungsgänge (z. B. Frankreich, Luxemburg, die Niederlande, Belgien), hauptsächlich durch On-the-job-Training am Arbeitsplatz (z.B. USA, Vereinigtes Königreich, Italien, Spanien) oder in der Form des dualen Systems (z. B. Deutschland, Österreich, Schweiz, Dänemark) organisieren. Das duale System hat gegenüber den anderen beiden Formen beruflicher Ausbildung im Globalisierungsprozess zunächst den Vorzug, dass für eine große Zahl von Jugendlichen der Übergang vom Schulsystem in das Beschäftigungssystem „planmäßiger“ und mit geringerer Arbeitslosigkeit verläuft. Die Kombination von theoretischem Lernen in der Berufsschule und praktischer Ausbildung am Arbeitsplatz schafft für die Jugendlichen eine institutionelle „Brücke“ zwischen dem Bildungssystem und den Firmen. Darüber hinaus sollte es für die Berufsanfänger von Vorteil sein, dass der Erwerb beruflicher Qualifikation überbetrieblich durch ein anerkanntes Zertifikat abgesichert ist. Denn diese Zertifikate können für die Arbeitskräfte und die Arbeitgeber beim Berufseinstieg und im späteren Berufsverlauf feste Bezugspunkte zur Bestimmung beruflicher Positionen darstellen. Der Nachteil des dualen Systems im Globalisierungsprozess scheint allerdings darin zu bestehen, dass es zu einer sehr engen Verkopplung von Ausbildungsab- 
schluss und Berufschance und damit zu einer hohen Starrheit und Abschottung beruflicher Mobilitätsprozesse kommt. Das unreglementierte und unzertifizierte On-the-job-Training und die rein schulische Berufsausbildung legen die Arbeitskräfte dagegen weniger durch soziale Definitionen auf enge berufliche Aufgabengebiete fest. Neue Generationen von Berufsanfängern werden in diesen beiden Systemen auch rascher an neu entstehende, zukunftsorientierte Berufstätigkeiten herangeführt („,hohe Generationenflexibilität“) als das beim dualen System der Fall ist, in dem immer wieder erst neue Ausbildungsberufe in einem langwierigen Aushandlungsprozess zwischen Arbeitgebern, Gewerkschaften und dem Staat geschaffen werden müssen. Auch kommt es in Ländern mit dualem System zu einer langfristigen, oft auch lebenslangen Ausgrenzung von „Ungelernten“, da diese ohne das Ausbildungszertifikat von qualifizierten Arbeitsmärkten praktisch ausgeschlossen werden. In Ländern mit On-the-jobTraining ist der Übergang von un- und angelernten Positionen zu qualifizierten Positionen dagegen eher fließend. Das heißt, im Zuge des Globalisierungsprozesses kommt es sowohl zu häufigeren Aufstiegen als auch zu vergleichsweise mehr Abstiegen und damit zu weniger „sozialer Sicherheit“ im Karriereverlauf. Schließlich steht das duale System im Zuge der Bildungsexpansion zunehmend vor dem Problem, dass sich im Verdrängungswettbewerb durch die jeweils Höherqualifizierten die Berufs- und Karrierechancen der „Praktiker“ immer mehr vermindern. Bei der Zuteilung von Planungs- und Leitungsfunktionen der Dienstleistungsklassen werden deswegen schulische und universitäre Bildungsabschlüsse immer mehr in den Vordergrund rücken. Damit wird die Lehre im Globalisierungsprozess, ähnlich wie in Frankreich, zunehmend zur Aufstiegssackgasse.

\subsection{Unterschiede der industriellen Beziehungen zwischen Kapital und Arbeit}

Die modernen Länder unterscheiden sich auch hinsichtlich der Art der industriellen Beziehungen zwischen Arbeit und Kapital auf der Ebene von Arbeitsorganisationen (z. B. der betrieblichen Mitbestimmung) und auf der Ebene nationaler Ökonomien (d. h. der Bedeutsamkeit nationaler Gewerkschaften und Arbeitnehmerorganisationen). Diese Unterschiede drücken sich in berufs-, sektoren- und länderspezifischen Verhandlungssystemen, Streikhäufigkeiten und administrativen Vereinbarungen (über Löhne, Arbeitsplatzsicherheit, Arbeitsbedingungen und Arbeitszeiten) aus.

In der Literatur werden die USA (und in jüngster Zeit auch das Vereinigte Königreich) als Beispiele für ein dezentralisiertes, dualistisches und auf Marktkräften beruhendes System industrieller Beziehungen genannt, in dem so genannte „offene“ Beschäftigungsverhältnisse dominieren. Das heißt, die Gewerkschaften sind dort schwach und dezentralisiert, so dass die Arbeitskräfte den globalen Unsicherheiten und Flexibilitätserfordernissen des Weltmarkts relativ ungeschützt ausgesetzt sind. Diese unmittelbare Konkurrenz sorgt dann (1) auf den meisten Arbeitsplätzen für vergleichsweise niedrige Löhne, (2) dafür, dass die Eintrittsprozesse der jungen Generation in den Arbeitsmarkt sich relativ fließend vollziehen, (3) für eine vergleichsweise hohe Berufsmobilität, (4) eine vergleichsweise kurze Dauer der Arbeitslosigkeit („Hire-and-fire-Prinzip“) und (5) eine relativ breite Verteilung prekärer Arbeitsverhältnisse auf die unterschiedlichsten Gruppen.

Auf der anderen Seite werden in der Literatur westeuropäische Länder als Arbeitsmärkte mit relativ „geschlossenen“ Beschäftigungsverhältnissen und zentralisierten Lohnsetzungsmechanismen klassifiziert. Schweden und Deutschland werden dabei in der Literatur als Beispiele für Länder mit besonders starken Gewerkschaften genannt und die 
südeuropäischen Länder wie Spanien und Italien gelten als Extremfälle für so genannte „Insider-Outsider-Arbeitsmärkte“. In diesen geschlossenen Arbeitsverhältnissen werden die meisten Beschäftigen vor den globalen Unsicherheiten und Flexibilitätserfordernissen des Weltmarkts relativ gut abgeschirmt und die Unsicherheiten und Risiken der Globalisierung werden überwiegend auf die Gruppen außerhalb des Arbeitsmarktes (die Berufsanfänger, Frauen nach der familiären Erwerbsunterbrechung oder die Arbeitslosen) abgewälzt. Das heißt, im Zuge des Globalisierungsprozesses entsteht in diesen Ländern eine Tendenz zu einer „neuen Unterklasse der Ausgeschlossenen“. Im Vergleich zu den Lebensbedingungen dieser „Ausgeschlossenen“ erhalten die Beschäftigten auf den internen Arbeitsmärkten relativ hohe Löhne. Die „geschlossenen Beschäftigungsverhältnisse“ führen dazu, dass (1) die Eintrittsprozesse der jungen Generation in den Arbeitsmarkt vor allem bei hoher allgemeiner Arbeitslosigkeit problematisch werden, (2) dass die Berufsmobilität vergleichsweise niedrig ausfällt, (3) dass die Dauer der Arbeitslosigkeit relativ lang ist und (4) dass sich die prekären Arbeitsverhältnisse (befristete Beschäftigungen, Teilzeitarbeit, Saisonarbeit etc.) stark auf diejenigen Gruppen konzentrieren, die einen Zugang zum Arbeitsmarkt suchen. In diesen „kollektiven“ Systemen haben insbesondere individuelle Ressourcen (wie soziale Herkunft, Ausbildung, Berufserfahrung etc.) eine geringere Auswirkung auf das Einkommen und die Karriereverläufe der bereits Beschäftigten.

\subsection{Verschiedene Wohlfahrtsstaatsregime}

In den modernen Ländern haben sich schließlich historisch auch unterschiedliche Wohlfahrtstaatsregime entwickelt, die mit sehr verschiedenen nationalen Vorstellungen sozialer Solidarität und unterschiedlich aktiven Bestrebungen zur Herstellung sozialer und geschlechtsspezifischer Chancengleichheit einhergehen. Die Unterschiede zwischen den Ländern drücken sich mit Blick auf die Mobilitätsprozesse insbesondere aus in (1) einer unterschiedlichen Priorität aktiver Arbeitsmarktpolitik zur Erlangung des Ziels der Vollbeschäftigung, (2) dem Umfang sozialstaatlicher (sog. ,dekommodifizierender“) Hilfen für diejenigen, die aus dem Arbeitsmarkt „,herausgefallen“ sind (Berufseinsteiger, Arbeitslose, Kranke, Sozialhilfeempfänger, Frauen in der Familienphase, Rentner etc.) und (3) in verschiedenen Anteilen der jeweils im staatlichen Sektor Beschäftigten.

Die USA, aber auch Kanada (und spätestens sein Margaret Thatcher das Vereinigte Königreich) gelten als Beispiele für „liberale Wohlfahrtsstaaten“, die durch eine passive Arbeitsmarktpolitik, moderate wohlfahrtsstaatliche Unterstützung Hilfsbedürftiger und einen vergleichsweise kleinen öffentlichen Beschäftigungssektor gekennzeichnet sind.

Norwegen, Dänemark und Schweden werden im Vergleich dazu häufig als Beispiele für das „sozialdemokratische Wohlfahrtsstaatsmodell“ genannt. Dort ist das Ziel der Arbeitsmarkt-, Steuer- und Sozialpolitik auf Vollbeschäftigung, Gleichheit von Männern und Frauen in Beruf und Familie und soziale Gleichheit hinsichtlich der Einkommensverteilung gerichtet. Das Vollbeschäftigungsziel wird dabei insbesondere durch eine Kombination von keynesianischer Nachfragepolitik und mobilitätsfördernden Maßnahmen wie Umschulung und Mobilitätshilfen sowie von befristeten Beschäftigungsverhältnissen zu erreichen versucht (sog. Flexicurity-Modell). Die hohe Vollzeitbeschäftigung der (verheirateten) Frauen beruht in diesen Wohlfahrtsstaaten auf der mit der Expansion wohlfahrtsstaatlicher Dienstleistungen (Kindergärten, Schulen, Krankenhäuser, Ganztagsschulen, Kindertagesstätten, Altenheime etc.) einhergehenden stark steigenden Beschäftigung im öffentlichen Sektor („Service for women creates jobs for women“) und zum Teil auf der hohen individuellen Steuerbelastung, 
die ein zweites Haushaltseinkommen erforderlich macht.

Deutschland und vor allem die Niederlande werden in der Literatur dagegen als „konservative Wohlfahrtsstaaten“ bezeichnet. Die Sozialpolitik dieser Staaten ist weniger auf aktive, beschäftigungsfördernde Maßnahmen gerichtet als vielmehr auf die wohlfahrtsstaatliche Absicherung derjenigen, die aus dem Arbeitsmarkt „herausgefallen“ sind (Arbeitslose, Berufseinsteiger, Kranke, allein erziehende Frauen, Rentner etc.) - obwohl sich hier durch „Hartz IV“ in der Schwerpunktsetzung in den letzten Jahren etwas geändert hat. Länder wie die Niederlande und Deutschland haben deswegen in der Vergangenheit Arbeitslose relativ lange finanziell unterstützt und großzügige Frühpensionierungssysteme entwickelt. Es handelt sich hier also um einen stark transferorientierten Wohlfahrtsstaatstyp, der weniger die Beschäftigungsrisiken durch aktive, keynesianischeArbeitsplatzbeschaffungsmaßnahmen zu bekämpfen versucht, sondern der durch sog. , ,dekommodifizierende“ Leistungen die nichterwerbstätige Bevölkerung abfedert. Dieser Wohlfahrtsstaatstyp zeichnet sich auch durch eine starke Verpflichtung aus, die Aufgaben der traditionellen Familie hinsichtlich Kindererziehung und Altenbetreuung aufrechtzuerhalten. Dementsprechend sind die wohlfahrtsstaatlichen Dienstleistungen (wie Kindergärten, Kindertagesstätten, Altenheime etc.) im Vergleich zum sozialdemokratischen Modell weit weniger stark ausgebaut und die Beschäftigung der (verheirateten) Frauen ist niedriger oder auf Teilzeitarbeit beschränkt (Blossfeld/Hakim 1997; Blossfeld/Drobnič 2001). In Deutschland trägt dazu auch das Ehegattensplitting bei, das Erwerbsunterbrechungen und Teilzeitarbeit von Ehefrauen privilegiert.

In den südeuropäischen Ländern wie Italien und Spanien dominiert schließlich ein eher „familienbezogener Wohlfahrtsstaat“. Hinsichtlich der staatlichen Wirtschafts- und Sozialpolitik ist er dem „,liberalen Wohlfahrtsstaat“ sehr ähnlich. Er zeichnet sich aber im Unterschied dazu durch eine starke ideologische und praktische Inanspruchnahme der Familien bei der Absicherung von Risiken aus. Dieses Modell basiert auf der in südeuropäischen Ländern soziokulturell noch tief verankerten Vorstellung, dass die Familie eine außerordentlich wichtige Institution der gegenseitigen Hilfe darstellt. Innerhalb der noch relativ traditionellen Familien wird diese Hilfe vor allem von Frauen geleistet, so dass deren Erwerbstätigkeit und Teilzeitarbeit im internationalen Vergleich außergewöhnlich niedrig ausfällt.

Schließlich sind noch die ehemals sozialistischen Länder zu erwähnen, die zwar alle einen gemeinsamen Startpunkt im Prozess der jüngsten Globalisierung haben (den sozialistischen oder kommunistischen Wohlfahrtsstaat), aber - wie sich empirisch zeigt - doch sehr unterschiedliche Wege der Integration in die Weltwirtschaft beschreiten. Einige dieser Länder, wie etwa Estland, haben sich für ein radikales marktliberales System entschieden, andere, wie etwa die Tschechische Republik oder Ungarn, bewegen sich eher auf einem weitgehend sozialdemokratischen Pfad.

\section{Das GLOBALIFE-Projekt}

Das GLOBALIFE-Projekt lieferte in der Projektlaufzeit von September 1999 bis Februar 2005 eine umfassende und in dieser Form bislang einzigartige Untersuchung der Auswirkungen des Globalisierungsprozesses auf individuelle Lebensverläufe von Menschen in verschiedenen OECD-Gesellschaften. In vier aufeinander folgenden Forschungsphasen analysierte das GLOBALIFE-Projekt in erwerbsbiografischer Reihenfolge die Auswirkungen des Globalisierungsprozesses auf vier zentrale Phasen im Lebens- und Erwerbsverlauf von Männern und Frauen: 
(1) den Übergang von der Jugend in das Erwachsenenalter und die während dieser Zeit stattfindende Etablierung im Arbeitsmarkt sowie deren Auswirkungen auf die Familienbildung und Fertilität,

(2) den Erwerbsverlauf von Männern in der Mitte ihrer beruflichen Karriere,

(3) den Erwerbsverlauf von Frauen, unter besonderer Berücksichtigung von Familienentwicklung und Mutterschaft,

(4) die späte Erwerbskarriere und den Übergang in den Ruhestand.

In jeder der vier Projektphasen wurden die Auswirkungen des Globalisierungsprozesses auf individuelle Lebensverläufe in insgesamt 17 verschiedenen modernen Gesellschaften international vergleichend untersucht. Auf Basis einer detaillierten Analyse ihrer institutionellen und kulturellen Charakteristika wurden diese Länder in fünf verschiedene „Wohlfahrtsregime“ eingeteilt:

Liberale Wohlfahrtsregime: Kanada, Großbritannien, USA;

Konservative Wohlfahrtsregime: Deutschland, die Niederlande, Frankreich;

Sozialdemokratische Wohlfahrtsregime: Norwegen, Dänemark, Schweden;

Familienorientierte Wohlfahrtsregime: Italien, Spanien, Irland, Mexiko;

Post-sozialistische Wohlfahrtsregime: Estland, Ungarn, Tschechische Republik, Polen. Das GLOBALIFE-Projekt umfasst damit ein ausgesprochen breites Spektrum von modernen Ländern unterschiedlichster institutioneller und kultureller Prägung, die die Auswirkungen des Globalisierungsprozesses auf individuelle Lebensverläufe sehr unterschiedlich gestalten. Neben der ausgesprochen großen Breite an untersuchten Ländern stellt jedoch auch die explizite Berücksichtigung der „post-sozialistischen“Länder Osteuropas in einer Globalisierungsstudie ein Novum in der international vergleichenden Sozialforschung dar.

Während der Projektlaufzeit arbeiteten insgesamt 22 Wissenschaftler unterschiedlicher Nationalität an den Universitäten Bielefeld und Bamberg als festangestellte Mitarbeiter des GLOBALIFE-Projektes. Gleichzeitig bestanden feste Kooperationsbeziehungen mit insgesamt 49 externen Wissenschaftlern aus 17 verschiedenen Ländern. Diese ausgewiesenen Sachverständigen und nationalen Experten erstellten in jeder der vier Forschungsphasen auf Basis repräsentativer Längsschnittdaten umfassende Länderberichte über die Auswirkungen des Globalisierungsprozesses im jeweiligen nationalen Kontext. Die Resultate dieser Forschungsarbeiten wurden im Rahmen spezieller, jährlich stattfindender internationaler Fachsymposien ausführlich diskutiert und in das umfassende GLOBALIFE-Forschungskonzept integriert.

Die Ergebnisse der vier Projektphasen wurden in vier Sammelbänden bei international renommierten sozialwissenschaftlichen Fachverlagen veröffentlicht: Blossfeld, Klijzing, Mills und Kurz (2005), Blossfeld, Mills und Bernardi (2006), Blossfeld und Hofmeister (2006) sowie Blossfeld, Buchholz und Hofäcker (2006).

\section{Ausgewählte Ergebnisse des GLOBALIFE-Projekts}

Aufgrund des knappen zur Verfügung stehenden Platzes kann hier nur ausführlicher auf die Ergebnisse der ersten Phase des GLOBALIFE-Projekts eingegangen werden (siehe Tabelle 1). Es ging dort um die Folgen der Globalisierung für die Lebenssituation junger Erwachsener beim Übergang vom Bildungs- in das Beschäftigungssystem, also die Phase der beruflichen Etablierung und Familiengründung (Blossfeld/Klijzing/Mills/Kurz 2005). In dieser Forschungsphase des GLOBALIFE-Projekts standen vor allem die folgenden Fragen im Vordergrund: Wie gelingt Jugendlichen und jungen Erwachsenen unter den Bedingungen 
der Globalisierung der Einstieg in den Arbeitsmarkt? Bewirkt Globalisierung durch die Flexibilisierung von Beschäftigungsverhältnissen eine Zunahme von Unsicherheiten beim Berufseinstieg? In welcher Weise beeinflussen länderspezifische Institutionen, wie etwa die Regulierung von Arbeitsmärkten oder die Gestaltung wohlfahrtsstaatlicher Leistungen, das Ausmaß von Unsicherheiten beim Berufseinstieg? Wie wirken sich veränderte berufliche Einstiegs- und Beschäftigungsmuster bei jungen Menschen auf deren familiäre Entscheidungen - wie z. B. das Eingehen von Partnerschaften oder die Gründung einer Familie - aus? Entwickeln junge Menschen unter den Bedingungen zunehmender Unsicherheit neue, alternative Entscheidungsstrategien?

Die empirischen Ergebnisse des GLOBALIFE-Projekts zeigen deutlich, dass im Zuge der Globalisierung in allen untersuchten Ländern vor allem junge Menschen mit massiv zunehmenden Unsicherheiten beim Einstieg in das Erwerbsleben konfrontiert werden (siehe Spalte 2 in Tabelle 1). Diese Unsicherheiten manifestieren sich insbesondere in Form einer starken Zunahme prekärer, atypischer Formen der Beschäftigung (z. B. zeitlich befristeter Beschäftigung, Teilzeitarbeit, prekärer Formen der Selbständigkeit oder gering angesehener Arbeiten) und geringerer Einkommen. Aufgrund dieser Entwicklungen können junge Menschen in der Übergangsphase vom Bildungs- in das Beschäftigungssystem als die „Verlierer“ des Globalisierungsprozesses angesehen werden.

Die Wirkungen des Globalisierungsprozesses auf die Arbeitsmarktpositionen der jungen Generation sind dabei je nach Wohlfahrtsstaats- und Arbeitsmarktregime sehr unterschiedlich. In den ausgeprägten Insider-Outsider-Märkten Südeuropas (aber auch z. T. in Deutschland) kommt es vermehrt zu Arbeitslosigkeit und/oder zeitlich befristeten Beschäftigungsverhältnissen in Südeuropa auch zusätzlich zum Anwachsen von prekärer Selbstständigkeit. In den Niederlanden kommt es zu vermehrten Teilzeitbeschäftigungen von jungen Männern und jungen Frauen; und in den offenen Beschäftigungsverhältnissen der liberalen Länder (USA, Großbritannien) manifestieren sich die Auswirkungen des Globalisierungsprozesses in wachsenden Einkommensverlusten bei den jungen Generationen.

Junge Menschen sind besonders stark von Globalisierung betroffen, weil ihnen vielfach die Berufserfahrung und eine stabile Verankerung im Arbeitsmarkt fehlen, insbesondere in „internen“ Arbeitsmärkten. Sie können oftmals nicht auf soziale Netzwerke zu Arbeitsorganisationen zurückgreifen und besitzen nicht die Verhandlungsmacht, stabile und kontinuierliche Arbeitsverhältnisse einzufordern. Ihre Arbeitsverträge können darüber hinaus vergleichsweise einfach veränderten Bedingungen angepasst, d. h., flexibilisiert und zu ihren Lasten verschlechtert werden.

Für junge Menschen wird Bildung im Globalisierungsprozess immer wichtiger. Besonders hart von den globalen Veränderungen werden Berufseinsteiger ohne Qualifikation getroffen. Globalisierung verstärkt insgesamt die sozialen Ungleichheiten innerhalb der jungen Generation, weil individuelle Ressourcen durch die zunehmende Relevanz des Marktes und der individuellen Konkurrenz an Bedeutung gewinnen.

Die Erfahrung von Unsicherheit im jungen Erwachsenenalter hat ihrerseits Konsequenzen für familiäre Entscheidungsprozesse. Steigende ökonomische und zeitliche Unsicherheiten (durch Teilzeitarbeit, Einkommensverluste, Arbeitslosigkeit, befristete Arbeitsverhältnisse) führen dazu, dass junge Menschen das Eingehen einer Partnerschaft und die Gründung einer Familie zunehmend aufschieben oder sogar völlig darauf verzichten (siehe Spalte 3 und 4 in Tabelle 1).

Eine bemerkenswerte Ausnahme stellt in diesem Zusammenhang Irland dar (siehe Spalte 2 in Tabelle 1). Der irische Fall ist nahezu ein „Bilderbuchbeispiel“ dafür, wie ein Land von 
Tab. 1: Wachsende Arbeitsmarktunsicherheit junger Erwachsener und ihr Einfluss auf die Familiengründung (Zusammenfassung der Ergebnisse der 13 GLOBALIFE-Länderstudien)

\begin{tabular}{|c|c|c|c|c|c|}
\hline \multicolumn{6}{|c|}{ Wachsende Unsicherheit bei jungen Erwachsenen } \\
\hline \multicolumn{6}{|c|}{ Wirkung der Beschäftigungsunsicherheit auf } \\
\hline \multirow[t]{2}{*}{ Wohlfahrtsstaatsregime } & \multirow{2}{*}{$\begin{array}{l}\text { Unsicherheit } \\
\text { der Arbeits- } \\
\text { marktposition } \\
\text { jüngerer } \\
\text { Kohorten }\end{array}$} & \multicolumn{2}{|c|}{ Partnerschaft } & \multicolumn{2}{|c|}{ Elternschaft } \\
\hline & & Männer & Frauen & Männer & Frauen \\
\hline \multicolumn{6}{|l|}{ Konservativ } \\
\hline Deutschland & $\uparrow$ & $\downarrow$ & $\uparrow$ & $\downarrow$ & $\uparrow$ \\
\hline Niederlande & $\uparrow$ & $\downarrow$ & $\uparrow$ & $\downarrow$ & $\uparrow$ \\
\hline Frankreich & $\uparrow$ & $\downarrow$ & $\uparrow / \leftrightarrow$ & $\downarrow$ & $\uparrow / \downarrow$ \\
\hline \multicolumn{6}{|l|}{ Sozialdemokratisch } \\
\hline Schweden & $\uparrow$ & $\downarrow / \leftrightarrow$ & $\downarrow / \leftrightarrow$ & $\downarrow$ & $\downarrow$ \\
\hline Norwegen & $\uparrow$ & $\downarrow$ & $\downarrow$ & $\downarrow$ & $\downarrow$ \\
\hline \multicolumn{6}{|l|}{ Post-sozialistisch } \\
\hline Ungarn & $\uparrow$ & $\downarrow$ & $\downarrow$ & $\downarrow$ & $\uparrow$ \\
\hline Estland* & $\uparrow$ & $\downarrow / \leftrightarrow$ & $\downarrow / \leftrightarrow$ & $\downarrow / \leftrightarrow$ & $\downarrow / \leftrightarrow$ \\
\hline \multicolumn{6}{|l|}{ Liberal } \\
\hline Vereinigtes Königreich & $\uparrow$ & $\begin{array}{c}\leftrightarrow(\mathrm{NL}) \\
\downarrow(\mathrm{H})\end{array}$ & $\begin{array}{l}\downarrow(\mathrm{NL}) \\
\leftrightarrow(\mathrm{H})\end{array}$ & $\leftrightarrow$ & $\uparrow$ \\
\hline Kanada & $\uparrow$ & $\downarrow$ & $\downarrow$ & $\downarrow$ & $\downarrow$ \\
\hline Vereinigte Staaten $q$ & $\uparrow$ & - & $\downarrow$ & - & $\downarrow$ \\
\hline \multicolumn{6}{|l|}{ Familienorientiert } \\
\hline Mexiko우 & $\uparrow$ & - & $\leftrightarrow$ & - & $\leftrightarrow$ \\
\hline Italien & $\uparrow$ & $\downarrow$ & $\uparrow$ & $\downarrow$ & $\uparrow$ \\
\hline Spanien & $\uparrow$ & $\downarrow$ & $\uparrow$ & $\downarrow$ & $\uparrow$ \\
\hline Irland & $\downarrow$ & - & $\leftrightarrow$ & - & $\leftrightarrow$ \\
\hline
\end{tabular}

Legende: $\uparrow=$ Anstieg der Rate, $\leftrightarrow=$ keine signifikate Veränderung, $\downarrow=$ Abnahme der Rate, - = nicht analysiert, $\phi=$ nur Frauen untersucht, $(\mathrm{H})=$ Heirat, $(\mathrm{NL})=$ nichteheliche Lebensgemeinschaften, * Analyse nicht nach Geschlecht getrennt möglich.

Globalisierung profitieren kann, indem es sich der Konkurrenz auf dem Weltmarkt stellt, offene Handelsbeziehungen fördert und steuerliche Anreize für ausländische Investoren anbietet. Irland, das Ende der neunziger Jahre auf seinem Arbeitsmarkt nahezu Vollbeschäftigung erreichen konnte, ist das einzige Land im Rahmen unserer Untersuchung, in dem die 
Globalisierung tatsächlich zu einer Abnahme von Unsicherheiten geführt hat. Seit Mitte der neunziger Jahre ist in Irland auch ein deutlicher Wiederanstieg der Heirats- und Geburtenraten zu beobachten. Der Konkurrenzvorteil Irlands gegenüber anderen modernen Industriestaaten ergibt sich aber aus der Tatsache, dass andere Länder ihre Unternehmenssteuern nicht so deutlich gesenkt haben. Der erfolgreiche Weg Irlands kann deswegen nicht von allen Ländern gleichzeitig beschritten werden, weil damit der Vorteil eines Sonderweges verloren ginge.

Als Reaktion auf steigende Unsicherheiten im Lebenslauf entwickelten junge Menschen vier verschiedene Verhaltens- und Anpassungsstrategien: (1) Langfristig bindende Entscheidungen werden zunehmend aufgeschoben, die Jugendphase wird immer mehr zu einem „Moratorium“ und Übergänge in das Erwerbsleben verlaufen oft chaotisch. (2) Junge Menschen weichen zunehmend in Alternativrollen zur Erwerbstätigkeit aus (sie bleiben z. B. länger im Bildungssystem anstatt sich als ,arbeitslos“ definieren zu lassen). (3) Es bilden sich zunehmend flexiblere Formen von Partnerschaften heraus (z. B. nicht-eheliche Lebensgemeinschaften), die eine Anpassung an die veränderten Bedingungen von Unsicherheit ohne das Eingehen langfristig bindender Versprechen ermöglichen. (4) Es entwickeln sich insbesondere in den familienorientierten Staaten Mittel- und Osteuropas geschlechtsspezifische Strategien des Umgangs mit Unsicherheit (siehe Spalten 3-6 in Tabelle 1): Männer sind in immer geringerem Maße in der Lage, als „Ernährer“ eine langfristige Einkommenssicherheit für einen Haushalts zu erbringen, so dass die Gründung eines eigenen Familienhaushalts von ihnen oft aufgeschoben wird. Unqualifizierte Frauen, die nichts zu verlieren haben, weichen hingegen als Reaktion auf wachsende Arbeitsmarktunsicherheiten teilweise in die Sicherheit der Familie und in die traditionellen Rollen der Mutter und Hausfrau aus. Umgekehrt hängt die Neigung hochqualifizierter Frauen, Kinder zu bekommen, in zunehmend unsichereren Arbeitsmärkten davon ab, dass sie ihre Berufschancen durch eine Vereinbarkeit von Familie und Beruf wahren können. Da die Kinderbetreuung in Südeuropa schlecht ausgebaut ist, entscheiden sich viele der qualifizierten Frauen für den Beruf.

Es ist ein paradoxes Ergebnis des Globalisierungsprozesses, dass gerade in familienorientierten Staaten die Geburtenrate aufgrund der zunehmenden Erfahrung von Unsicherheiten deutlich sinkt (siehe die Spalten 5 und 6 in Tabelle 1). Im Gegensatz zu demografischen Ansätzen, die diesen Wandel auf einen „Wertewandel“ in modernen Gesellschaften zurückführen, ist eine an zunehmenden Unsicherheiten orientierte Globalisierungserklärung in der Lage, das Paradox zwischen einem oftmals ausgeprägten Kinderwunsch und dessen tatsächlicher Nicht-Realisierung bei jungen Erwachsenen aufzulösen: Die Gründung einer Familie erfordert ein Mindestmaß an wirtschaftlicher und sozialer Zukunftssicherheit, die unter den globalen Bedingungen zunehmender Arbeitsmarktunsicherheit häufig nicht gewährleistet werden kann. Der Verzicht auf Kinder ist somit eine ökonomisch und sozial rationale Reaktion der einzelnen Individuen auf strukturelle Entwicklungen. Das zurückhaltende Fertilitätsverhalten zeigt sich in jüngster Zeit auch stark in den Transformationsländern Osteuropas.

Demgegenüber ist die Geburtenrate in den skandinavischen Ländern, in denen der Staat für junge Menschen und Familien vergleichsweise großzügige, universelle Leistungen und Betreuungseinrichtungen für Kinder zur Verfügung stellt und eine aktive Beschäftigungspolitik betreibt, deutlich höher.

Wichtig ist es in diesem Zusammenhang darauf hinzuweisen, dass es nicht auf das absolute Unsicherheitsniveau ankommt, sondern auf das in einem Land von den Arbeitskräften jeweils subjektiv erlebte relative Unsicherheitsniveau. Die jungen Leute vergleichen sich im Alltag in jedem Land mit ,signifikanten Anderen“ (wie Freunden, Verwandten, Bekannten, beruflichen Vorbildern), wenn sie ihre individuelle Arbeitsmarktlage beurteilen. So ist beispielsweise in 
den USA das absolute Unsicherheitsniveau für die junge Generation durch Berufsmobilität insgesamt weit höher als in vielen europäischen Ländern. Es kommt dort häufiger zu Hireand-Fire-Prozessen, aber die Arbeitslosen können darauf vertrauen, dass sie rasch wieder einen anderen Job finden können, also zum „Insider“ werden. Arbeitsmarktunsicherheit, Berufsmobilität und Flexibilität haben dort deswegen auch eine andere soziale Bedeutung und werden subjektiv anders wahrgenommen als in den Insider-Outsider-Märkten Europas, in denen das „Outsider-Sein“ oft einen identitätsgefährdenden, dauerhaften Ausschluss von der Arbeit bedeutet und flexible Beschäftigungsverhältnisse in der Regel nur als eine Behelfs- und Übergangslösung zu einer dauerhaften Beschäftigung betrachtet werden. Junge Menschen in flexibilisierten Beschäftigungsformen werden deswegen in den europäischen Insider-Outsider-Märkten ihr Schicksal als gravierend negativer erfahren als in den USA. Der sich im Zuge der Globalisierung vollziehende Übergang von einem Insider-Outsider-Arbeitsmarkt zu einem flexiblen Arbeitsmarkt wird deswegen von der jungen Generation nicht nur als schmerzvoller erlebt, sondern wird sich auch über längere Zeiträume hinziehen - bis sich in den Strukturen des nationalen Arbeitsmarktes die flexibleren Beschäftigungsverhältnisse in voller Breite als Standardbeschäftigungsform etabliert haben.

\section{Literatur}

Blossfeld, Hans-Peter, Sandra Buchholz, Dirk Hofäcker (2006): Globalization, uncertainty and late careers in society. London

Blossfeld, Hans-Peter, Sonja Drobnič. (2001): Careers of contemporary societies. A cross-national comparison of the transition from male breadwinner to dual-earner families. Oxford

Blossfeld, Hans-Peter, Catherine Hakim (1997): Between equalization and marginalization. Part-time working women in Europe and the United States of America. Oxford

Blossfeld, Hans-Peter, Heather Hofmeister (2006): Globalization, uncertainty and women's careers: An international comparison. Cheltenham, UK/Northampton, MA, USA

Blossfeld, Hans-Peter, Melinda Mills, Erik Klijzing \& Karin Kurz (2005): Globalization, uncertainty and youth in society. London

Blossfeld, Hans-Peter, Melinda Mills, Fabrizio Bernardi (2006): Globalization, uncertainty and men's careers: An international comparison. Cheltenham, UK/Northampton, MA, USA

Dreher, Axel (2005): Does globalization affect growth? Evidence from a new index of globalization, Manuskript KOF, ETH Zürich (im Erscheinen)

Anschrift des Verfassers:

Prof. Dr. Hans-Peter Blossfeld

Otto-Friedrich-Universität Bamberg

Fakultät Sozial- und Wirtschaftswissenschaften

Lehrstuhl für Soziologie I

Postfach 1549

D-96045 Bamberg

hans-peter.blossfeld@sowi.uni-bamberg.de

\section{Schlagwörter: Arbeitsmarkt der jungen Generation, Fertilitätsrückgang, Flexibilisierung, Globalisierung, Unsicherheit}

